\title{
Periodontically Accelerated Osteogenic Orthodontics-A Review
}

\author{
MN. Prabhu ${ }^{1, *}$, J. Sabarinathan ${ }^{2}$ \\ ${ }^{1}$ Department of periodontics, pidc, Penang, Malaysia \\ ${ }^{2}$ Department of orthodontics, pidc \\ *Corresponding author: prabhumds@rediffmail.com
}

Received August 23, 2013; Revised August 26, 2013; Accepted December 03, 2013

\begin{abstract}
It is well known that most of the orthodontic treatments require more time for completion of the treatment. This technique literally accelerates the orthodontic corrections. The appliance is worn by the patient before the surgery. After the periodontal surgery, force is applied to the appliance which moves the tooth faster. In this technique Distraction osteogenesis \& Regional Accelerated phenomenon are combined to produce a faster orthodontic correction as desired by the Orthodontist. The concept behind the technique, the pros and cons, and the technique as well are reviewed in this article.
\end{abstract}

Keywords: corticotomy, osteopenia, osteotomy, flap, tissue remodeling

Cite This Article: MN. Prabhu, and J. Sabarinathan, "Periodontically Accelerated Osteogenic Orthodontics-A Review.” American Journal of Biomedical Research 1, no. 4 (2013): 132-133. doi: 10.12691/ajbr-1-4-9.

\section{Introduction}

The orthodontic tooth movement is based on biology of the bone, technique of the orthodontist and the materials used. It usually takes a year or more to get a complete orthodontic correction done depending on the severity of the case. When surgery was involved in orthodontic management the treatment duration is reduced further. The concept of Periodontally Accelerated Osteogenic Orthodontics further accelerated the orthodontic movement thereby reducing the treatment time. It was L.C. Bryan in 1893 who first introduced Corticotomy-facilitated tooth movement1. The current corticotomy procedures adopted or modified by most clinicians are based on Heinrick Köle's combined radicular corticotomy/supraapical osteotomy technique, first described in 1959.

\section{Concept}

Periodontally Accelerated Osteogenic Orthodontics treatment modality is a combination of Distraction osteogenesis \& Regional Accelerated phenomenon. It's a combination of selective decortications which facilitated orthodontic technique and alveolar augmentation. In simple words it combines Periodontics and Orthodontics in treating the patient. Growth of new bone by surgically distracting the bone is the concept behind this technique. It is well known that there was a direct correlation between bone injury \& the intensity of its healing response. The temporary burst of localized soft \& hard tissue remodeling occurring following the regional accelerated phenomenon rebuilds bone back to its normal shape [2].
Following corticotomy reduced mineralization of alveolar bone occurs which leads to rapid tooth movement. The bone is decorticated in Periodontally Accelerated Osteogenic Orthodontics, unlike corticotomies where it is cut and removed. This is the difference between Periodontally Accelerated Osteogenic Orthodontics, and corticotomy. By decortications it is meant that some external surface of the bone is removed. The bone then goes through a phase called osteopenia, where the mineral content is decreased temporarily. Due to the release of rich deposits of calcium by the tissue of the alveolar bone, new bone begins to mineralize in about 20-55 days. This enables the orthodontist to move the teeth 2 to 3 times faster than the time required in traditional orthodontic therapy. Patients who have undergone Periodontally Accelerated Osteogenic Orthodontics are with the appliances for about four to ten months. The retainers are given following that for upto six months [2].

\section{Procedure}

The following are the steps involved:

\subsection{Role of Orthodontist}

Orthodontic treatment before surgery.

Appliances are put on a few days before the Periodontally Accelerated Osteogenic Orthodontics procedure.

\subsection{Role of Periodontist}

Under Local anesthesia, incisions are given and the flap is reflected. Usually a full thickness flap is reflected. Esthetic factors are to be considered while raising the flap. Vertical incisions are to be made at least one tooth away from the area to be decorticated. Decortication lines and 
points are made for upto $0.5 \mathrm{~mm}$ depth. Selective medullary penetration should also be made to enhance bleeding. The decortications consist of removing the cortical part of the bone alone without involving the cancellous bone ${ }^{3}$. Round bur is used for this purpose. If needed bone grafts mixed with antibiotics can be given accordingly. Flaps are put back in place and sutures are given. Sutures should be in place for a period of two weeks.

\subsection{Post Operative Instructions}

The patient is put on soft diet.

Antibiotics, analgesics and mouth rinses are given.

To reduce the post-operative swelling ice packs are given immediately after surgery. Necessary medications can also be given as per the requirement. Recovery takes 7-10 days.

\subsection{Role of Orthodontist}

Orthodontic treatment after surgery.

The orthodontic force should be applied immediately after the surgery, as it was found to be the best time to initiate the movement [4]. But some prefer to start the tooth movement two weeks after the surgery. The orthodontist adjusts the appliances once in every two weeks. Depending upon the individual cases, the orthodontic correction will be over in four to ten months[1,5]. These procedures were found to reduce the treatment time required for the correction of Crowding and they accelerate canine retraction after premolar extraction. Molar intrusions and open bite corrections can also be carried out at a faster pace with this technique.

\section{Modifications}

It is not always necessary to carry out the periodontal surgical procedure for the full mouth. The surgical procedure can be confined to the site where it is needed as determined by the orthodontist [6,7]. Also the surgical procedure can be split into two procedures and can be carried out in two separate appointments to reduce the complication of necrosis [8]. It can be used to expedite the rate of movement of individual teeth or dental segments.

\section{Contraindications}

1. Poor Periodontal status; but it had been reported that a Class II malocclusion case with severe bi maxillary protrusion and adult periodontitis have been successfully treated [9].

2. Root damage.

3. Class III malocclusion cases

\subsection{Advantage $[3,10]$}

1. Appliances are worn for a lesser time by the patient.

2. The need for extractions is reduced.

3. When compared to traditional techniques chances of root resorption is less.

4. Tooth movement is faster.

5. Safer Expansion of Constricted Arches

6. Except for severe Class III skeletal dysplasia, Periodontally Accelerated Osteogenic Orthodontics can replace orthognathic surgery to the extent as determined by the Orthodontist.
7. There is an increase in alveolar bone volume after orthodontic treatment; the amount of bone formation around the tooth in its new position is moreindicates a gain in additional strength in long term when compared to traditional techniques. This also gives a better facial profile in some cases.

\subsection{Disadvantages}

1. Risks of surgery including interdental bone loss and loss of attached gingiva.

2. Subcutaneous hematomas of the head and neck, Pain. [11].

3. Cost factor.

\section{Conclusion}

As the density of bone / the amount of bone required to be remodeled by orthodontic force is less there happens a faster movement of the teeth which reduces the whole treatment time after periodontal surgery. The whole treatment time can be reduced to less than a year for completion of the treatment. The results obtained were the same.

In this fast paced world this is "Fast Paced Orthodontics" successfully offered by the Orthodontist and Periodontist for the benefit of those who want a faster change in profile.

\section{References}

[1] Goyal Amit 1, Kalra JPS. Periodontally accelerated osteogenic orthodontics (PAOO) - a review. J Clin Exp Dent 2012; 4(5):e 292-6.

[2] M. Thomas Wilcko, William M. Wilcko. An Evidence-Based Analysis of Periodontally Accelerated Orthodontic and Osteogenic Techniques: A Synthesis of Scientific Perspectives. Seminars in Orthodontics December 2008; 14 (4):305-316.

[3] Ali H Hassan. Corticotomy-Assisted Orthodontic Treatment: Review. Open Dent J. 2010; 4: 159-164.

[4] Eric Jein-Wein Liou. Rapid orthodontic tooth movement into newly distracted bone after mandibular distraction osteogenesis in a canine model. American Journal of Orthodontics and Dentofacial Orthopedics April 2000; 117 (4): 391-398.

[5] Leena Palomo .Salient Periodontal Issues for the Modern Biologic Orthodontist. Seminars in Orthodontics December 2008;14 (4): 229-245.

[6] Hessam Nowzari. Periodontally Accelerated Osteogenic Orthodontics Combined with Autogenous Bone Grafting. Compendium May 2008; 29(4):1-9.

[7] M. Thomas Wilcko, DMD, William M. Wilcko. Accelerated Osteogenic Orthodontics Technique: A 1-Stage Surgically Facilitated Rapid Orthodontic Technique With Alveolar Augmentation. Journal of Oral and Maxillofacial Surgery October 2009; 67(10): 2149-2159.

[8] Kyu-Rhim Chung . Speedy Surgical Orthodontic Treatment With Skeletal Anchorage in Adults-Sagittal Correction and Open Bite Correction. Journal of Oral and Maxillofacial Surgery October 2009; 67(10), 2130-2148.

[9] Seong-Hun Kim. Severe bimaxillary protrusion with adult periodontitis treated by corticotomy and compression osteogenesis.Korean J Orthod Feb 2009; 39(1):54-65.

[10] Ali Saad Thafeed AlGhamdi . Corticotomy facilitated orthodontics: Review of a technique. The Saudi Dental Journal January 2010; 22(1):1-5.

[11] Oztürk M, Doruk C, Ozeç I, Polat S, Babacan H, Biçakci AA. Pulpal blood flow: effects of corticotomy and midline osteotomy in surgically assisted rapid palatal expansion. J Craniomaxillofac Surg. 2003; 31:97-100. 